\title{
Effects of Grain Shape Genes Editing on Appearance Quality of Erect-Panicle Geng/ Japonica Rice
}

Ting Mao ${ }^{1,2+}$, Mingdong Zhu ${ }^{1,3 \dagger}{ }^{\dagger}$ Zhonghua Sheng ${ }^{1}$, Gaoneng Shao', Guiai Jiao', Amos Musyoki Mawia', Shakeel Ahmad ${ }^{1}$, Lihong Xie ${ }^{1}$, Shaoqing Tang ${ }^{1}$, Xiangjin Wei ${ }^{1}$, Shikai Hu ${ }^{1 *}$ (D) and Peisong Hu${ }^{1 *}$

\begin{abstract}
Long grain geng/japonica rice has a higher market preference due its excellent appearance quality. The dense and erect-panicle 1 (dep 1) gene has been widely used in the breeding of high-yielding geng/japonica rice cultivars in China. However, this gene causes short and round grain shape thus making it less attractive in global rice markets. Therefore, breeding of high-yielding long-grain geng/japonica rice cultivars by incorporating dep 1 with major-effect grain shape gene is of high priority in rice industry. Up to now, multiple grain shape genes' effect mechanism has been clearly elaborated, however, under the background of erect-panicle geng/japonica rice, the effect of major grain shape genes on the appearance quality need to be further clarified, as detailed reports are limited. Utilizing CRISPR/ Cas9 technology, a series of near-isogenic lines (NILs) (YF47 ${ }^{\text {dep } 1}$-gw8, YF47 $7^{\text {dep } 1}$-gs3, YF4 $7^{\text {dep } 1}$-gl7, YF $47^{\text {dep } 1}$ - $q g / 3$ and YF47 ${ }^{\text {dep } 1}$-tgw6) in Yanfeng 47(YF47 ${ }^{\text {dep } 1}$ ) background were created. Grain appearance and yield components analysis showed that: (1) All NILs' grain length to width ratio was significantly increased compared to that of YF47dep1, excepted YF47 dep 1_gs3, (2) The chalkiness degree was significantly reduced in all of the NILs, (3) In all of the NILs, YF47 ${ }^{\text {dep } 1}$-gw8 grains exhibited the greatest length to width ratio and the lowest chalkiness degree, (4) The composition of glume cells and filling characteristics of the endosperm were two key factors contributing grain shape and grain chalk variations, respectively, and $\mathbf{v}$ ) Owning to a substantial increase in the thousand grain weight, the yields of YF47 ${ }^{\text {dep } 1}$-gs 3 and YF47 ${ }^{\text {dep } 1}$-tgw6 were significantly higher than that of YF47 $7^{\text {dep } 1}$, whereas $Y F 47^{\text {dep } 1}$ - $q g / 3$ exhibited the lowest yield because of a dramatic decrease in the effective panicle number and thousand grain weight. All the results revealed that pyramiding dep 1 with major-effect grain shape alleles was an effective approach to improving the appearance quality of erect-panicle geng/japonica rice, owning to both of the appearance quality and yield improvement, GS3 and TGW6 alleles can be applied directly for breeding long-grain shape geng/japonica rice, and editing GW8 resulted in excellent appearance quality but low yield, therefore, this gene would be difficult to use directly but can be considered as the core germplasm resource.
\end{abstract}

Keywords: Rice, Grain shape gene, Appearance quality, Gene editing, Molecular design breeding

\footnotetext{
*Correspondence: hushikai@caas.cn; hupeisong@caas.cn

${ }^{\dagger}$ Ting Mao and Mingdong Zhu have contributed equally to this work.

'State Key Laboratory of Rice Biology, China National Center for Rice Improvement, China National Rice Research Institute, Hangzhou 310006,

China

Full list of author information is available at the end of the article
}

\section{Findings}

With the structural adjustment and upgrading of the rice industry, the market demand for superior quality geng/ japonica rice is increasing every year (Chen et al. 2018). The grain shape of traditional geng/japonica rice in China is mostly short and round, but recently long grain shape has become preferred by the market because of its excellent appearance quality (Xu and Chen 2016; Huang and 
Qian 2017). Currently, the dep1 gene is widely used in the breeding of geng/japonica rice in China due to highyielding population characteristics, however, the gene also brings in characteristics of short grains and low thousand grain weight (Liu et al. 2018). Therefore, breeding of high-yielding long-grain geng/japonica rice cultivars by incorporating dep 1 with major-effect grain shape genes is of high priority in rice industry, as the theoretical basis and required germplasm reports are limited (Chen et al. 2012; Xu and Chen 2016).

Multiple genes for grain shape have been cloned, some of which have strong effects and wide applications, including the major-effect genes controlling grain length, GS3, qGL3 and GL7/GW7, the thousand grain weight gene TGW6, plant shape gene DEP1 (Fan et al. 2006; Zhang et al. 2012; Ishimaru et al. 2013; Wang et al. 2015a, b) and major-effect genes that control grain width, GW2, GW5, GS5 and GW8 (Song et al. 2007; Wan et al. 2008; Li et al. 2011; Wang et al. 2012). Multiple studies have shown that the grain shape genes directly determine the shape of rice grains by regulating the development of glume cells (Fan and Li 2019), and also indirectly affect grain chalk by determining endosperm development after fertilization (Wang et al. 2008; Li et al. 2014). In addition, a series of endogenous hormones and starch synthesisrelated enzymes are also involved in the dynamic development of the glumes and endosperm. For example, brassinosteroids (BR) and indole acetic acid (IAA) are reportedly related to the development of glume cells ( $\mathrm{Li}$ et al. 2018), whereas the enzyme activities of adenosine diphosphate glucose (ADPG), granule-bound starch synthase (GBSS), soluble starch synthase (SSS) and starch branching enzyme (SBE) on endosperm development is significant (Dong et al. 2008).

In the present study, utilizing CRISPR/Cas9 technology, we created a series of NILs (YF47 ${ }^{d e p 1}-g w 8$, YF47 $7^{d e p 1}$-gs3, YF47 $7^{d e p 1}-g l 7, \quad$ YF $47^{d e p 1}-q g l 3$ and $\mathrm{YF}^{2} 7^{\text {dep } 1}$-tgw6) in YF $47^{\text {dep } 1}$ background. Further, the comparison of grain appearance and yield were contracted, and the related impact factors were also deeply clarified. The objectives of this study included: (1) Clarifying the effects of $G W 8$, GS3, GL7, qGL3, and TGW6 genes (partial major-effect grain shape genes) on grain appearance and yield of erect panicle rice, (2) creating a series of long-grain erectpanicle geng/japonica rice gremplasm, and evaluating its breeding application value in aspect of appearance quality and yield, and (3) providing the theoretical basis for breeding long-grain shape geng/japonica rice.

We analyzed YF47 $7^{\text {dep } 1}$ and 96 geng/japonica rice varieties' genotype distribution at the GW8, GS3, GL7, qGL3 and TGW6 loci, which were widely used in xian/ indica rice breeding programs. As shown in Additional file 3: Fig. S1 and Additional file 2: Table S1, YF47 ${ }^{\text {dep } 1}$ was classified as short and round grain type ("Nipponbare" type) on both of the above-mentioned locus, and also, very few long grain type alleles have been used in geng/japonica rice breeding in northern China. Therefore, by utilizing CRISPR/Cas9 technology, we created a series of NILs as our research materials, which carried the mutant alleles at the above loci under the background of geng/japonica rice cultivar, YF47 $7^{\text {dep } 1 \text {, having }}$ an erect panicle architecture. The NILs comprised of YF $47^{d e p 1}-g w 8$, YF $47^{d e p 1}-g s 3$, YF $47^{d e p 1}-g l 7$, YF $47^{d e p 1}-q g l 3$ and YF4 $7^{d e p 1}-\operatorname{tg} w 6$, which present single base insertion in target site and led to truncated proteins of various sizes compared with YF47 ${ }^{d e p 1}$ (wild type, WT) (Fig. 1a). Besides the number of tillers, the plant architecture of NILs had insignificant differences compared with YF47 $7^{\text {dep } 1}$ (Fig. 1b).

The grain lengths and widths were determined at maturity stage (Fig. $2 \mathrm{a}-\mathrm{d}$ ), the grain length of YF47 $7^{\text {dep } 1}-g w 8$ was $5.29 \mathrm{~mm}$ and around $5.1 \mathrm{~mm}$ for the other NILs, which showed significant increase compared to that of $Y F 47^{d e p 1}(4.9 \pm 0.08 \mathrm{~mm})$. The grain width was significantly lower in YF47 ${ }^{d e p 1}-g w 8(2.39 \pm 0.09 \mathrm{~mm})$ and significantly higher in YF47 ${ }^{d e p 1}-g s 3(2.90 \pm 0.06 \mathrm{~mm})$, compared to YF47 ${ }^{\text {dep } 1}(2.80 \pm 0.09 \mathrm{~mm})$. No significant differences in width were observed in the other lines. The average length to width ratio of YF47 $7^{\text {dep } 1}$ was 1.74 (Fig. 2e), but significantly higher in YF47 ${ }^{\text {dep } 1}-g w 8$ $(2.22 \pm 0.11), \quad$ YF $47^{d e p 1}-g l 7 \quad(1.81 \pm 0.11), \quad Y_{F} 47^{d e p 1}-q g l 3$ $(1.84 \pm 0.04)$, and YF47 ${ }^{d e p 1}$-tgw6 $(1.82 \pm 0.05)$. The length to width ratio of the $\mathrm{YF} 47^{\text {dep } 1}$-gs3 grain was statistically the same as that of YF47 $7^{\text {dep } 1}$ due to the increase in its width. Scanning electron microscope analysis showed that the mean length of the glume cells in all of the NILs was significantly greater than that of YF47 ${ }^{d e p 1}$, with those of YF47 $7^{\text {dep } 1}$-gw 8 being the longest (Fig. $2 \mathrm{f}-\mathrm{i}$ ). The cell width in YF47 $7^{d e p 1}$-gw8 was significantly reduced but substantially increased in YF47 $7^{\text {dep } 1}-g s 3$, and no significant difference was observed in the other NILs. The cell number in YF47 ${ }^{d e p 1}$-gw8 and YF47 $7^{d e p 1}$-gl7 was significantly increased, whereas it decreased dramatically in YF47 $7^{\text {dep } 1}$-gs3 and YF47 ${ }^{\text {dep } 1}$-tgw6.

It has previously been reported that increased levels of IAA and BR might increase fruit size (Li et al. 2018). Therefore, we analyzed the levels of the endogenous hormones IAA and BR during the most vigorous period of glume development (Fig. 2j-k). Of all the NILs, no significant change was seen in YF47 $7^{d e p 1}-g l 7$ and YF47 ${ }^{d e p 1}-q g l 3$. However, the levels of IAA and BR were substantially reduced and elevated in YF47 $7^{d e p 1}-g w 8$ and YF $47^{d e p 1}$-tgw6, respectively. The IAA level was significantly elevated in YF47 ${ }^{\text {dep } 1}-g s 3$, whereas its level of BR remained statistically the same although slightly elevated. Along with the thousand grain weight (TGW) performance in Table 1, 


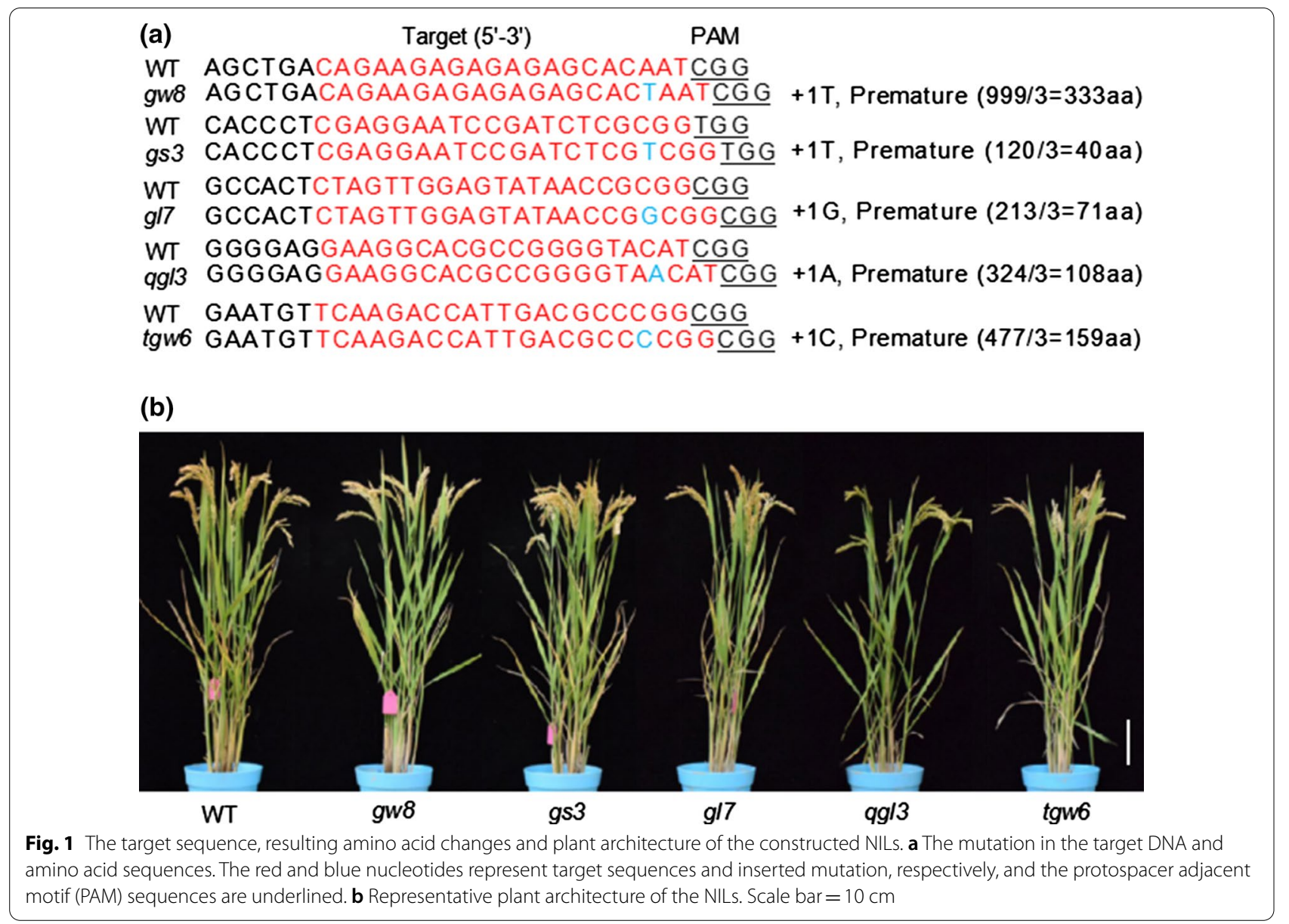

we speculated that IAA and BR might promote the TGW by regulating glume development. In erect-panicle varieties, short and round grains with low TGW are prone to form because of the up-regulated expression of $D E P 1$ (Sun et al. 2018). In our study, apart from YF47 ${ }^{d e p 1}-g w 8$, the level of dep1 expression was down-regulated in all of the transgenic plants (Fig. 2l), which may serve as a contributing factor to their elongated grain lengths. On the contrary, the level of $d e p 1$ was increased in $\mathrm{YF}_{4} 7^{d e p 1}{ }_{-}-g w 8$, which may be related to its significantly decreased TGW (Table 1).

The grain chalky characteristics of the NILs were investigated after the grains were processed into polished rice (Fig. 3a, b). Chalkiness degree in all the NILs was significantly lower than that of YF47 $7^{\text {dep } 1}$, which demonstrated an average of $7.31 \%$. The lowest level of chalkiness degree was observed in YF47 $7^{d e p 1}-g w 8$ with a value of $1.51 \pm 0.41 \%$, followed by YF47 ${ }^{\text {dep } 1}$-qgl3 $(2.28 \pm 0.57 \%)$ and YF47 $7^{d e p 1}-g l 7(3.29 \pm 0.51 \%)$. The chalkiness degree of YF47 $7^{\text {dep } 1}$-gs3 and YF4 $7^{\text {dep } 1}$ - $\operatorname{tgw} 6$ was relatively high, measured as $5.37 \pm 1.19 \%$ and $4.08 \pm 0.40 \%$, respectively. Also, the length to width ratio and chalkiness degree showed a significant negative correlation (Fig. 3c). This indicated that increasing the length to width ratio of grains and reducing chalkiness degree were harmonized in the erect-panicle background.

To further investigate the effect of endosperm development on grain chalkiness variations, we analyzed the grain filling rate by calculating the changes in endosperm weight during 0-35 days after flowering (Fig. 3d, e). The maximum and average grain filling rates for all the NILs were significantly reduced compared to that of YF4 ${ }^{d e p}$. Of these, YF47 ${ }^{d e p 1}-g w 8$ showed the lowest maximum and average grain filling rates, whereas the measurements for YF $47^{d e p 1}-g s 3$ and YF4 $7^{d e p 1}-\operatorname{tgw} 6$ were relatively high. Wang et al., (2012) demonstrated that the activities of ADPG, GBSS, SSS and SBE are closely related to the filling rate of endosperm. Furthermore, we analyzed the activity changes in the starch biosynthesis-related enzymes in the NILs. Compared to YF47 ${ }^{d e p 1}$, the activities of these enzymes were significantly reduced in all of the NILs (Fig. $3 \mathrm{f}-\mathrm{i}$ ), with the exception that the activity of GBSS in YF47 ${ }^{d e p 1}$ - $g s 3$ and YF47 $7^{d e p 1}$ - $\operatorname{tg} w 6$ was substantially elevated. Of all the NILs, the activity levels of the 


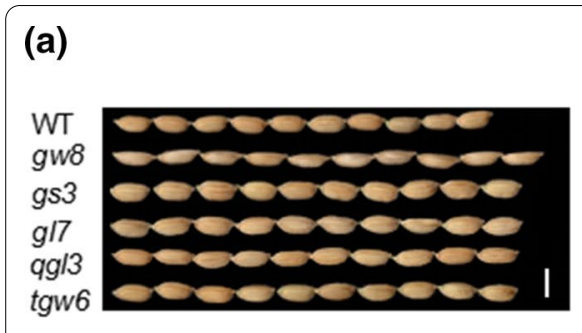

(e)

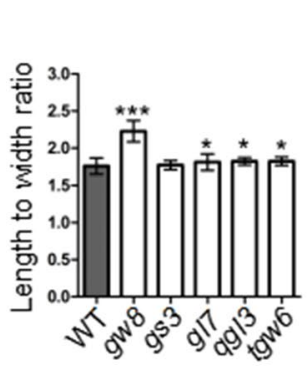

(h)

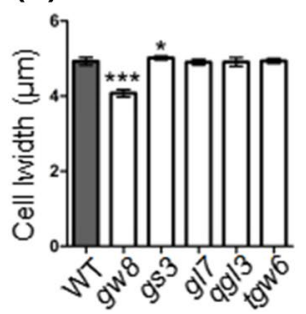

(f)

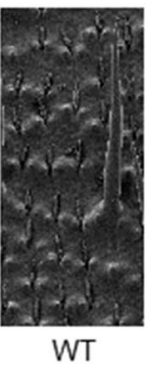

(i)

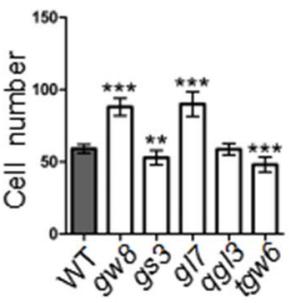

(b)

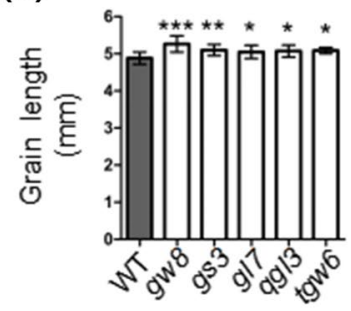

(c)

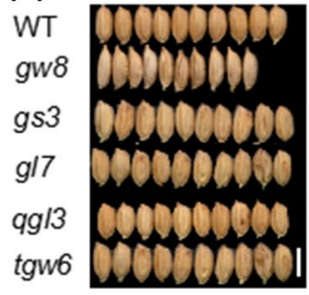

(d)

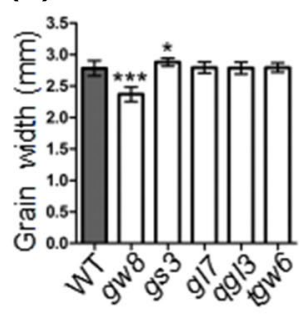

(g)

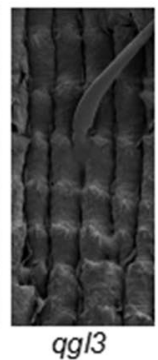

(k)
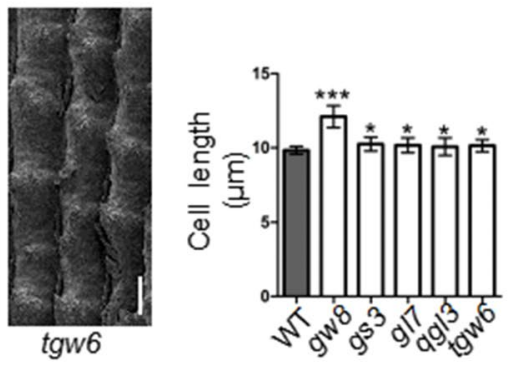

(I)
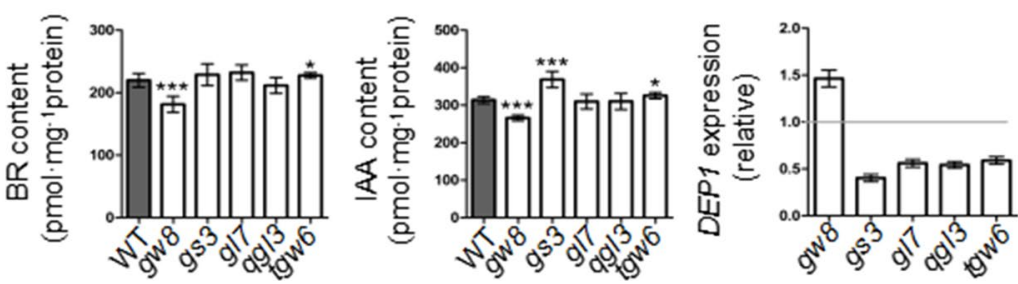

Fig. 2 The appearance and determination of grain shape and related impact factors analysis in YF47 ${ }^{\text {depl }}$ (WT) and the NILS. a, b The appearance (a) and comparison (b) of grain length in WT and NILs. Scale bar $=5 \mathrm{~mm}$. The data represent the mean $\pm s d,{ }^{* * *} P<0.001,{ }^{*} P<0.01,{ }^{*} P<0.05$, the same as below. c, d The appearance (c) and comparison (d) of grain width in WT and NILs. Scale bar $=5 \mathrm{~mm}$. e The comparison of length to width ratio in WT and NILs. $\mathbf{f}-\mathbf{i}$ The appearance $(\mathbf{f})$ and comparison of cell length $(\mathbf{j})$, cell width $(\mathbf{h})$ and number of cells (i) on the outer surface of the glumes in WT and the NILs. Scale bar $=10 \mu \mathrm{m}$. j, k The comparison of IAA (j) and BR (k) levels in YF47 ${ }^{\text {depl }}$ (WT) and the NILs. IThe relative expression of DEP1 in the NILS

Table 1 The comparison of yield traits in WT and the NILS

\begin{tabular}{|c|c|c|c|c|c|c|c|c|}
\hline \multirow[t]{2}{*}{ Varieties } & \multicolumn{2}{|l|}{ NEP } & \multicolumn{2}{|l|}{ NFGP } & \multicolumn{2}{|l|}{ TGW (g) } & \multicolumn{2}{|l|}{ Yield (kg ha $\left.{ }^{-1}\right)$} \\
\hline & 2019 & 2020 & 2019 & 2020 & 2019 & 2020 & 2019 & 2020 \\
\hline YF47 dep1 (WT) & $18 \pm 1.62$ & $18 \pm 1.15$ & $132 \pm 3.92$ & $131 \pm 2.12$ & $25.9 \pm 0.51$ & $25.8 \pm 0.65$ & $10,132.84 \pm 99.25$ & $9860.48 \pm 97.87$ \\
\hline YF47 ${ }^{\text {dep } 1}-g w 8$ & $19 \pm 1.58$ & $18 \pm 0.87$ & $128 \pm 4.15^{* *}$ & $125 \pm 3.33^{* *}$ & $23.2 \pm 0.48^{* *}$ & $23.1 \pm 0.38^{* *}$ & $9418.59 \pm 112.14^{* *}$ & $9168.47 \pm 112.14^{* *}$ \\
\hline YF47 $7^{\text {dep } 1-g s 3 ~}$ & $18 \pm 1.34$ & $18 \pm 1.36$ & $125 \pm 2.98^{* *}$ & $123 \pm 3.15^{* *}$ & $27.5 \pm 0.39^{* *}$ & $27.6 \pm 0.29^{* *}$ & $10,777.61 \pm 86.51^{* *}$ & $10,494.13 \pm 115.21^{* *}$ \\
\hline YF47 ${ }^{\text {dep } 1-g l 7 ~}$ & $19 \pm 1.87$ & $17 \pm 0.97$ & $127 \pm 3.14^{* *}$ & $129 \pm 2.97^{*}$ & $24.4 \pm 0.42^{* *}$ & $24.3 \pm 0.42^{* *}$ & $9610.35 \pm 79.24^{* *}$ & $9287.97 \pm 96.21^{* *}$ \\
\hline YF47 ${ }^{\text {dep } 1}$-qg/3 & $16 \pm 0.98^{* *}$ & $15 \pm 1.15^{* *}$ & $131 \pm 3.61$ & $127 \pm 3.45^{*}$ & $23.9 \pm 0.48^{* *}$ & $23.9 \pm 0.61^{* *}$ & $9085.29 \pm 96.51^{* *}$ & $8992.38 \pm 97.89^{* *}$ \\
\hline YF47 ${ }^{\text {dep } 1-t g w 6 ~}$ & $18 \pm 1.12$ & $19 \pm 1.71$ & $128 \pm 3.28^{*}$ & $120 \pm 4.12^{* *}$ & $26.7 \pm 0.54^{* *}$ & $26.6 \pm 0.42^{* *}$ & $10,774.82 \pm 115.41^{* *}$ & $10,402.42 \pm 93.21^{* *}$ \\
\hline
\end{tabular}

NEP, number of effective panicles; NFGP, number of filled grains per panicle; TGW, thousand grain weight; ${ }^{*} P<0.01,{ }^{*} P<0.05$

four enzymes in YF47 ${ }^{d e p 1}$-gw8 were the lowest. These results indicated that the up-regulation of these four enzymes was helpful in increasing the filling rate, and the relatively high activity of GBSS in YF47 $7^{\text {dep } 1}-g s 3$ and
YF $47^{d e p 1}-\operatorname{tg} w 6$ might be due to the high endosperm dry weight and high TGW.

The variation in yield traits of the NILs was compared in two consecutive growing seasons (Table 1). Generally, 
(a)

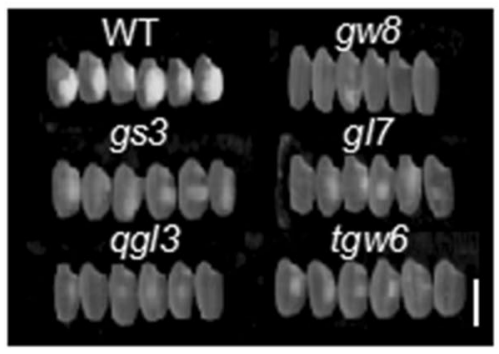

(d)

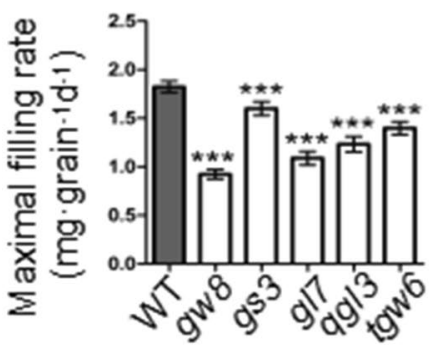

(g)

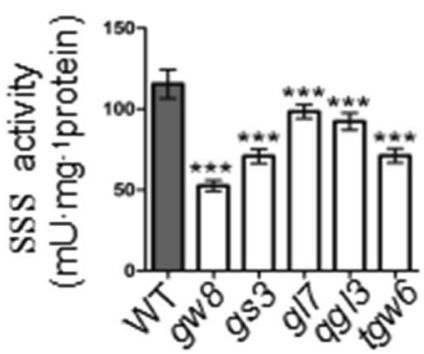

(b)

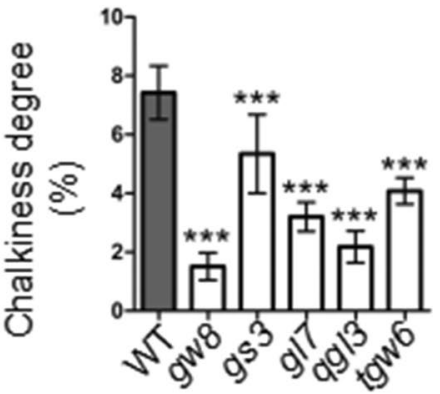

(e)

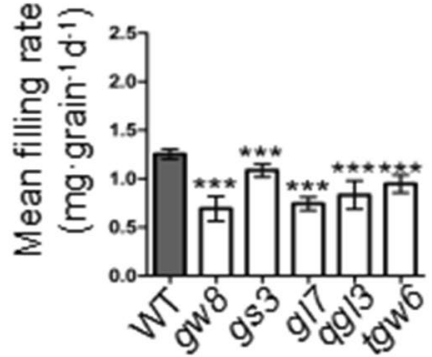

(h)

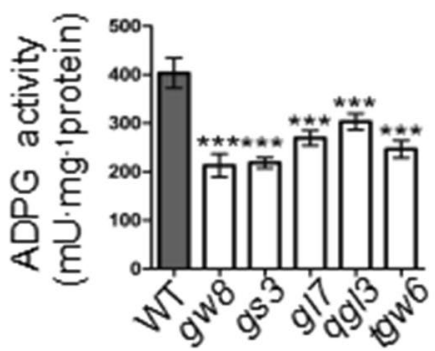

(c)

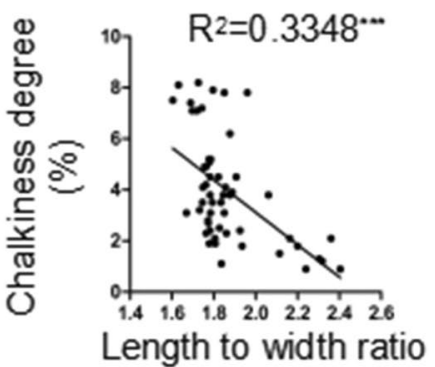

(f)

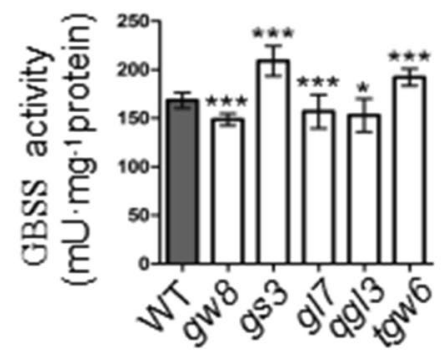

(i)

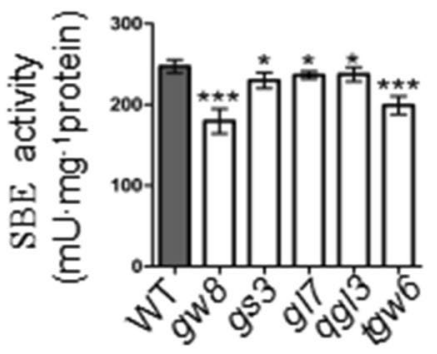

Fig. 3 The appearance and determination of grain chalk and related impact factors analysis in YF47 dep1 (WT) and the NILs. a, b The appearance (a) and comparison (b) of chalkiness degree in WT and the NILs. Scale bar $=5 \mathrm{~mm}$. c The correlation analysis of the length to width ratio and chalkiness degree in YF47 ${ }^{\text {dep } 1}$ and the NILs. d, e The maximum (d) and average (e) grain filling rates in WT and the NILs. f-i The comparison of ADPG content (f), GBSS content (g), SSS content (h) and SBE content (i) in WT and the NILs during endosperm development

the yields of YF4 $7^{\text {dep } 1}$-gs3 and YF4 $7^{\text {dep } 1}$-tgw6 improved significantly compared to the control YF47 ${ }^{\text {dep } 1}$ due to the substantially increased TGW, whereas YF47 ${ }^{\text {dep } 1}$-qgl3 exhibited the lowest yield due to the significantly reduced effective panicle number and TGW. However, the yields of YF47 ${ }^{\text {dep } 1}-g w 8$ and $\mathrm{YF}^{2} 7^{\text {dep } 1}-g l 7$ were relatively low owing to the dramatically decreased TGW and grain number per panicle.

While ensuring the high yield of erect-panicle varieties, the primary breeding goal is to develop long grain geng/japonica rice with the desired appearance qualities via the genetic improvement of grain shape (Xu and Chen 2016; Huang and Qian 2017). As pioneer research establishments, the China National Rice Research Institute (CNRRI) and Jiaxing Academy of Agricultural Sciences have developed Jiahe 218 and Jiahe 212 with a grain length to width ratio of 3.0 through the aggregation of dep 1 and gs3 (Huang and Qian 2017), which provides a reference for the genetic improvement of grain shape in erect-panicle varieties. However, the Jiahe series rice varieties contain a large amount of tropical japonica background (Huang and Qian 2017), which is different from our research basing on the temperate japonica background. In the present study, YF4 $7^{\text {dep } 1}$-gw 8 grain exhibited the greatest length to width ratio of 2.2, comparing the best-known long 
grain high quality japonica rice varieties (the length to width ratio is generally more than 2.5) (Huang and Qian 2017), the grain shape of NILs in this article is still not ideal. We speculated that the simultaneous pyramiding of GS3/TGW6 and GW8 long type allele should be an effective way of developing erect-panicle geng/japonica rice with excellent appearance quality and high yield.

In summary, our results demonstrated that editing of grain shape genes was an effective approach to creating long-grain erect-panicle geng/japonica rice germplasm. Owning to both of the appearance quality and yield improvement, GS3 and TGW6 alleles can be applied directly for breeding long-grain shape geng/japonica rice, and editing GW8 resulted in excellent appearance quality but low yield. Therefore, this gene would be difficult to use directly but can be considered as the core germplasm resource. All this work could provide the required germplasm and theoretical basis for breeding of high-yielding long-grain geng/japonica rice cultivars.

\begin{abstract}
Abbreviations
dep1: dense and erect-panicle 1; NILs: Near isogenic lines; YF47 dep1: Yanfeng 47; BR: Brassinosteroids; IAA: Indole acetic acid; ADPG: Adenosine diphosphate glucose; GBSS: Granule-bound starch synthase; SSS: Soluble starch synthase; SBE: Starch branching enzyme; WT: Wild type; NEP: Number of effective panicles; NFGP: Number of filled grains per panicle; TGW: Thousand grain weight.
\end{abstract}

\section{Supplementary Information}

The online version contains supplementary material available at https://doi. org/10.1186/s12284-021-00517-5.

\section{Additional file 1. Materials and Methods}

Additional file 2: Supplemental Table 1. The distribution of genotype on GW8, GS3, GL7, qGL3 and TGW6 derived from 96 rice germplasms in Liaoning province of China.

Additional file 3: Supplemental Fig. 1. Genotype analysis of GW8, GS3, GL7, qGL3 and TGW6 on YF47 ${ }^{\text {dep } 1}$ and 96 rice germplasms.

Additional file 4: Supplemental Fig. 2. The gene structures, target sequences for editing genes and vector map.

Additional file 5: Supplemental Table 2. Primers used to analyze target sequences and identify vectors.

Additional file 6: Supplemental Table 3. Primers used for Real-time PCR.

\section{Acknowledgements}

Not applicable.

\section{Authors' contributions}

TM and SH designed and performed the experiments, and analyzed data; $Z \mathrm{~S}$, GS, GJ, AMM, MZ, SA, LX, ST and XW participated in NILs construction and data analysis; $\mathrm{PH}, \mathrm{SH}$ and TM wrote the manuscript and prepared the illustrations. $\mathrm{PH}$ and $\mathrm{SH}$ conceived the idea and supervised the project. All authors read and approved the final manuscript.

\section{Funding}

This work was supported by grants from the National Natural Science Foundation of China (32071991, 31872861 and 31972961), the Key Research and Development Program of Zhejiang Province (2021C02056-1), the International Science \& Technology Innovation Program of Chinese Academy of Agricultural
Sciences (CAASTIP, CAAS-ZDRW202109), China National Rice Research Institute Key Research and Development Project (CNRRI-2020-01), the National Key Research and Development Program of China (2020YFE0202300), the Central Public-interest Scientific Institution Basal Research Fund (No.CPSIBRFCNRRI-202103), and the PhD research startup foundation of Liaoning Province (2020-BS-300).

\section{Availability of supporting data}

The data sets supporting the results of this article are included within the article and its additional files.

\section{Declarations}

Ethical approval and consent to participate

Not applicable.

\section{Consent for publication}

Written informed consent for publication was obtained from all participants.

\section{Competing interests}

The authors declare that they have no competing interests.

\section{Author details}

${ }^{1}$ State Key Laboratory of Rice Biology, China National Center for Rice Improvement, China National Rice Research Institute, Hangzhou 310006, China.

${ }^{2}$ Liaoning Institute of Saline-Alkali and Utilization, Panjin 124010, China.

${ }^{3}$ Hunan Rice Research Institute, Changsha 410125, China.

Received: 9 June 2021 Accepted: 5 August 2021

Published online: 10 August 2021

\section{References}

Chen WF, Xu ZJ, Tang L (2012) Advances and prospects of rice breeding for super high yield in China. J Shenyang Agric Univ 43(06):643-649 (in Chinese)

Chen MJ, Liu GF, Yu H, Wang B, Li JY (2018) Towards molecular design of rice plant architecture and grain quality. Chin Sci Bull 63(14):1275-1289 (in Chinese)

Dong MH, Zhao BH, Wu XZ, Chen T, Yang JC (2008) Difference in hormonal content and activities of key enzymes in the grains at different positions on a rice panicle during grain filling and their correlations with rice qualities. Scientia Agricultura Sinica 41(2):370-380 (in Chinese)

Fan YW, Li YB (2019) Molecular, cellular and Yin-Yang regulation of grain size and number in rice. Mol Breed 39:163

Fan CC, Xing YZ, Mao HL, Lu TT, Han B, Xu CG, Li XH, Zhang QF (2006) GS3, a major QTL for grain length and weight and minor QTL for grain width and thickness in rice, encodes a putative transmembrane protein. Theor Appl Genet 112(6):1164-1171

Huang HY, Qian Q (2017) Progress in genetic research of rice grain shape and breeding achievements of long-grain shape and good quality japonica Rice. Chin J Rice Sci 31(6):665-672 (in Chinese)

Ishimaru K, Hirotsu N, Madoka Y, Murakami N, Hara N, Onodera H, Kashiwagi T, Ujiie K, Shimizu Bl, Onishi A, Miyagawa H, Katoh E (2013) Loss of function of the IAA-glucose hydrolase gene TGW6 enhances rice grain weight and increases yield. Nat Genet 45(6):707-711

Li YB, Fan CC, Xing YZ, Jiang YH, Luo LZ, Sun L, Shao D, Xu CJ, Li XH, Xiao JH, He YQ, Zhang QF (2011) Natural variation in GS5 plays an important role in regulating grain size and yield in rice. Nat Genet 43(12):1296-1269

Li YB, Fan CC, Xing YZ, Yun P, Luo LJ, Yan B, Peng B, Xie WB, Wang GW, Li XH, Xiao JH, Xu CG, He YQ (2014) Chalk5 encodes a vacuolar $\mathrm{H}^{+}$-translocating pyrophosphatase influencing grain chalkiness in rice. Nat Genet 46(4):398-404

Li N, Xu R, Duan PG, Li YH (2018) Control of grain size in rice. Plant Reprod 31:237-251

Liu Q, Han RX, Wu K, Zhang JQ, Ye YF, Wang SS, Chen JF, Pan YJ, Li Q, Xu XP, Zhou JW, Tao DY, Wu YJ, Fu XD (2018) G-protein $\beta$ y subunits determine grain size through interaction with MADS-domain transcription factors in rice. Nat Commun 9:852 
Song XJ, Huang W, Shi M, Zhu MZ, Lin HX (2007) A QTL for rice grain width and weight encodes a previously unknown RING-type E3 ubiquitin ligase. Nat Genet 39:623-630

Sun SY, Wang L, Mao HL, Shao L, Li XH, Xiao JH, Ouyang YD, Zhang QF (2018) A G-protein pathway determines grain size in rice. Nat Commun 9:851

Wan XY, Weng JF, Zhai HQ, Wang JK, Lei CL, Liu XL, Guo T, Jiang L, Su N, Wan JM (2008) Quantitative Trait Loci (QTL) Analysis for rice grain width and fine mapping of an identified QTL allele $g w-5$ in a recombination hotspot region on Chromosome 5. Genetics 179(4):2239-2252

Wang E, Wang JJ, Zhu XD, Hao W, Wang LY, Li Q, Zhang LX, He W, Lu BR, Lin HX, Ma H, Zhang GQ, He ZH (2008) Control of rice grain-filling and yield by a gene with a potential signature of domestication. Nat Genet 40(11):1370-1374

Wang SK, Wu K, Yuan QB, Liu XY, Zheng BL, Lin XY, Zeng RZ, Zhu HT, Dong GJ, Qian Q, Zhang GQ, Fu XD (2012) Control of grain size, shape and quality by OsSPL 16 in rice. Nat Genet 44(8):950-954

Wang SK, Li S, Liu Q, Wu K, Zhang JQ, Wang SS, Wang Y, Chen XB, Zhang Y, Gao CX, Wang F, Huang HX, Fu XD (2015a) The OsSPL16-GW7 regulatory module determines grain shape and simultaneously improves rice yield and grain quality. Nat Genet 47(8):949-954

Wang $Y X$, Xiong GS, Hu J, Jiang $L, Y u H, X u J$, Fang $Y X$, Zeng $L$, Xu RB, Xu J, Ye WJ, Meng XB, Liu RF, Chen HQ, Jing YH, Wang YH, Zhu XD, Li JY, Qian Q (2015b) Copy number variation at the GL7 locus contributes to grain size diversity in rice. Nat Genet 47(8):944-948

Xu ZJ, Chen WF (2016) Research progress and related problems on japonica. Super rice in northern China. Scientia Agricultura Sinica 49(2):239-250 (in Chinese)

Zhang XJ, Wang JF, Huang J, Lan HX, Wang CL, Yin CF, Wu YY, Tang HJ, Qian Q, Li JY, Zhang HS (2012) Rare allele of OSPPKL1 associated with grain length causes extra-large grain and a significant yield increase in rice. Proc Natl Acad Sci 109(52):21534-21539

\section{Publisher's Note}

Springer Nature remains neutral with regard to jurisdictional claims in published maps and institutional affiliations.

\section{Submit your manuscript to a SpringerOpen ${ }^{\circ}$ journal and benefit from:}

- Convenient online submission

- Rigorous peer review

- Open access: articles freely available online

- High visibility within the field

- Retaining the copyright to your article

Submit your next manuscript at $\boldsymbol{\nabla}$ springeropen.com 